\title{
Pulmonary benign metastasising leiomyoma: a single-institution case series
}

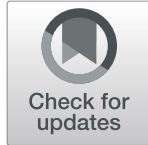

Karishma Chandarana ${ }^{1 *}\left(\mathbb{D}\right.$, Edward J. Caruana ${ }^{1}$ (D) Cathy J. Richards ${ }^{2}$, Sridhar Rathinam ${ }^{1}$ (D) and Apostolos $\operatorname{Nakas}^{1}$ (D)

\begin{abstract}
Background: Benign metastasising leiomyoma (BML) is a rare and often asymptomatic presentation of smooth muscle tumour of uterine origin, occurring outside the uterus. We present the first case series of pulmonary BML in a contemporary UK population.

Patients were identified from prospective histopathological databases spanning from 2003 to 2019. Data was collected retrospectively from physical and electronic data sources.

Results: Six post-menopausal females were identified with an average age of $44 \pm 8$ years (mean \pm SD). Fifty percent of our cohort was asymptomatic with an incidental finding of pulmonary nodules, whilst the others complained of non-specific respiratory symptoms. Five patients (88\%) had multiple lesions (median 9, range 2 to 12) - with bilateral distribution, measuring an average of $11 \mathrm{~mm}$ (range 7 to 27) in size on cross-sectional imaging. All patients underwent diagnostic surgical pulmonary wedge resection, with no perioperative morbidity.

Immunohistochemistry confirmed the presence of 'spindle cells', staining positive for smooth muscle actin, desmin and progesterone receptors for all patients (100\%), and oestrogen receptors in five patients (87\%). Four patients (67\%) underwent a hysterectomy prior to diagnosis of BML and one (17\%) due to the diagnosis itself. Four patients (67\%) underwent oophorectomy, whilst one patient required hormonal suppression therapy. Survival was $100 \%$ at a median follow-up of 59.5 months (range 29 to 166).

Conclusions: BML is a rare clinical entity accounting for a small proportion of patients presenting with pulmonary nodules. Following successful tissue diagnosis, outcomes with conservative or medical management are excellent.
\end{abstract}

Keywords: Pulmonary nodule, Benign, Oncology, Thoracic surgery, Metastatic, Women's health

\section{Background}

Multiple pulmonary nodules remain a challenging prospect with regards to management. The differential diagnosis varies from benign entities to disseminated metastasis. Benign metastasising leiomyoma (BML), first described by Steiner et al. in 1939 [1], is a smooth muscle tumour of primary uterine origin, metastasising to a distant location within the body. With approximately 150 cases reported in the literature, there is limited knowledge surrounding this condition. The largest contribution of patients comes from two single-centre

\footnotetext{
* Correspondence: karishma.chandarana@nhs.net

${ }^{1}$ Department of Thoracic Surgery, Glenfield Hospital, University Hospitals of Leicester Trust, Leicester LE3 9QP, UK

Full list of author information is available at the end of the article
}

case series, published from continental Europe and North America [2, 3].

Extra-uterine metastases are commonly pulmonary; however, there have been numerous reports of BML in other organs [4]. The mode of dissemination remains uncertain; BML is more common in post-menopausal females who have undergone uterine surgery, suggesting surgery creates a haematogenous route for tumour spread $[5,6]$.

Microscopically, the tumour is formed of spindle cells showing no atypia, necrosis, mitosis or invasion [7]. However, uterine tissue located distant to the original location suggests BML is a benign tumour with malignant potential, a low-grade slow-growing leiomyosarcoma [7, 8]. It has also been suggested that BML may display similarities with endometriosis [7]. 
Diagnosis is through tissue biopsy, wherein microscopic histopathology shows smooth muscle proliferation within lung parenchyma, and immunohistochemistry identifies smooth muscle actin, desmin and caldesmin. The presence of oestrogen and progesterone receptors and the ability for the nodules to change in size confirm hormonedependent growth $[4,9]$.

Presentation is often an incidental finding of 'pulmonary nodule(s)' on radiological imaging (Fig. 1), but patients can present with a wide variety of respiratory symptoms, prompting radiological imaging of the chest. Although rare, there are few known cases of a direct relationship between the nodules and subsequent symptoms due to close proximity with vital structures or respiratory failure [4].

Hysterectomy removes the primary source of uterine leiomyoma, with bilateral oophorectomy eliminating endogenous hormone source promoting nodule growth by surgical castration. Medical therapies such as gonadotropin-releasing hormone $(\mathrm{GnRH})$ analogues, luteinizing hormone-releasing hormone (LHRH) agonists or aromatase inhibitors offer hormonal manipulation to reduce tumour size. For asymptomatic patients with no evidence of progression, treatment often involves 'watchful waiting' [4].

BML is an uncommon and often overlooked diagnosis. Our study aims to help clinicians recognise BML in the differential diagnosis of pulmonary nodules and describe the implications for these patients across specialties.

\section{Methods}

Six patients with a diagnosis of BML were identified using histopathological data from electronic records.

Retrospective data collection was undertaken at a single tertiary university hospital over a 17-year period

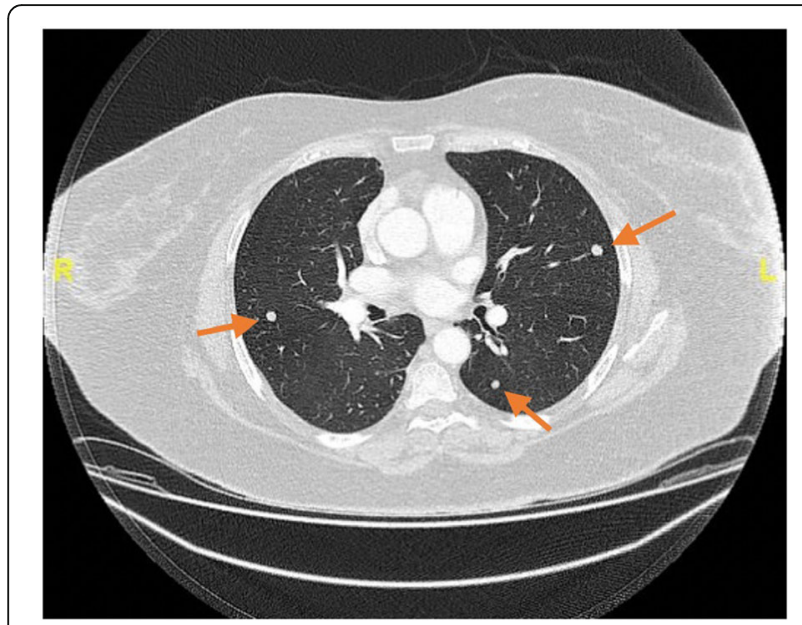

Fig. 1 Cross-sectional imaging demonstrating bilateral pulmonary nodules, later identified on histology as pulmonary benign metastasising leiomyoma
(2003-2019). In line with local and national governance in the UK, the study was registered as a clinical audit. Additional clinical data was collected and analysed from patient case notes and imaging.

\section{Results}

Patient demographics

Our study identified six patients, all post-menopausal females who were diagnosed with pulmonary BML at a median age of 45 years (range 38 to 56). Four patients were Caucasian (67\%) and two of Asian origin (33\%).

Three patients had undergone hysterectomy for symptomatic uterine leiomyoma prior to diagnosis (50\%), with the diagnosis of pulmonary BML prompting subsequent hysterectomy and oophorectomy in one patient (17\%). Patient demographics and presentations are summarised in Table 1.

\section{Presentation}

BML was an incidental finding in asymptomatic patients during routine imaging for two patients and in one patient with ureteric colic. Another three patients presented with respiratory symptoms, prompting plain imaging of the chest.

\section{Radiological imaging}

Five patients had multiple lesions, with bilateral distribution of spread (83\%). The median number of lesions was 9 (range 2-12), with a median lesion size of $11 \mathrm{~mm}$ (range 7 to 27). Description of the lung nodules on radiological imaging can be found in Table 2 .

\section{Pathway}

Following the radiological diagnosis of pulmonary nodules, patients were placed on a pathway through an established multidisciplinary team meeting formed of respiratory physicians, thoracic surgeons, pathologists, radiologists and medical oncologists. Following individual case discussion, all patients underwent a pulmonary wedge resection as a diagnostic procedure.

\section{Surgical procedure}

Five procedures were right sided (83\%), and one procedure was left sided (17\%).

A posterolateral thoracotomy in the left 5 th intercostal space was performed for the first patient, as the lesion in the left lower lobe was significantly larger than any of the other pulmonary nodules and the timing of the procedure (2005) was prior to an established VATS programme at our centre.

Video-assisted thoracoscopic surgery (VATS) on the right side was used as standard approach for the remaining five patients (83\%). 
Table 1 Patient demographics and presentation

\begin{tabular}{llllll}
\hline Patient $(\boldsymbol{n})$ & Gender & Hysterectomy & Oophorectomy & Symptoms at presentation & Age at BML diagnosis \\
\hline 1 & Female & No & No & Asymptomatic & 50 \\
2 & Female & Yes & No & Cough & 54 \\
3 & No & No & Pleuritic chest pain & 40 \\
4 & Female & Yes & Asymptomatic & 40 \\
5 & Female & Yes & Yes & Shortness of breath & 56 \\
6 & Female & Yes & Yes & Asymptomatic & 38 \\
\hline
\end{tabular}

\section{Histopathology}

Haematoxylin and eosin (H\&E) stained positive for spindle cells in all six samples (100\%) (Figs. 2 and 3). All six samples were positive for desmin and smooth muscle actin $(100 \%)$, with three samples also positive for caldes$\min (50 \%)$.

Biopsies confirmed the presence of progesterone receptors in six patients (100\%), oestrogen receptors in five patients (87\%).

\section{Follow-up}

Median length of stay was 2.5 days (range 1 to 8 ). No patients experienced any postoperative complications with $100 \%$ survival at median follow-up at 59.5 months (range 29 to 166).

Follow-up imaging was performed for all of our cohort. The choice of imaging modality and duration was largely subjective and dependant on the patient's lead clinician. For five patients, the nodules remained unchanged. One individual, who underwent a hysterectomy without oophorectomy, required adjuvant hormonal therapy with anastrozole to stabilise growth of pulmonary nodules in close proximity to hilar structures.

All patients remained under follow-up with interval imaging. As no established management protocol for patients with BML exists, three patients remain under the care of thoracic surgery, one patient under clinical oncology, and two under gynaecology oncology.

\section{Discussion}

Steiner et al. first described benign metastasising leiomyoma as a rare smooth muscle tumour of uterine origin found at a distant location in the body [1]. A fundamental pathological difference between a benign and malignant tumour is the ability to invade surrounding tissue [10], creating much uncertainty around the term 'benign metastasising leiomyoma'.

The growth of BML lesions is driven by hormonal influence due to the presence of oestrogen and progesterone receptors on the tumour surface. This can influence the clinical course and presentation. Most commonly, BML affects post-menopausal females, and presentation varies from asymptomatic to life threatening compression of vital structures within the thorax. Immunohistochemistry of the biopsied tissue confirms smooth muscle tumour, with no malignant features and positive staining of oestrogen and progesterone receptors [4].

Once BML is diagnosed in asymptomatic patients, management is individualised and often involves watchful waiting and interval imaging. In symptomatic patients or in those with nodules increasing in size, surgical oophorectomy or medications such as GnRH analogues and anti-oestrogens can slow or halt growth completely [4].

Similar to the literature [6], all our study participants were post-menopausal females through natural ageing or induced surgically by hysterectomy. Four (66\%) patients had a hysterectomy due to symptomatic uterine leiomyoma, with $50 \%$ of those having an additional oophorectomy. Whilst only $50 \%$ of our cohort were asymptomatic, those presenting with respiratory symptoms were due to co-existing pathology prompting imaging and not a causal relationship.

BML typically demonstrates variable uptake of fluorodeoxyglucose (FDG) intake on positron emission tomography (PET) [11, 12]; however, none of our patients

Table 2 Description of pulmonary nodules on imaging

\begin{tabular}{llll}
\hline Patient $(\boldsymbol{n})$ & Number of nodules & Bilateral distribution & Maximum diameter $(\mathbf{m m})$ \\
\hline 1 & 1 & No & 27 \\
2 & 12 & Yes & 14 \\
3 & 3 & Yes & No preoperative CT \\
4 & 9 & Yes & 11 \\
5 & 12 & Yes & 7 \\
6 & 2 & Yes & 8 \\
\hline
\end{tabular}




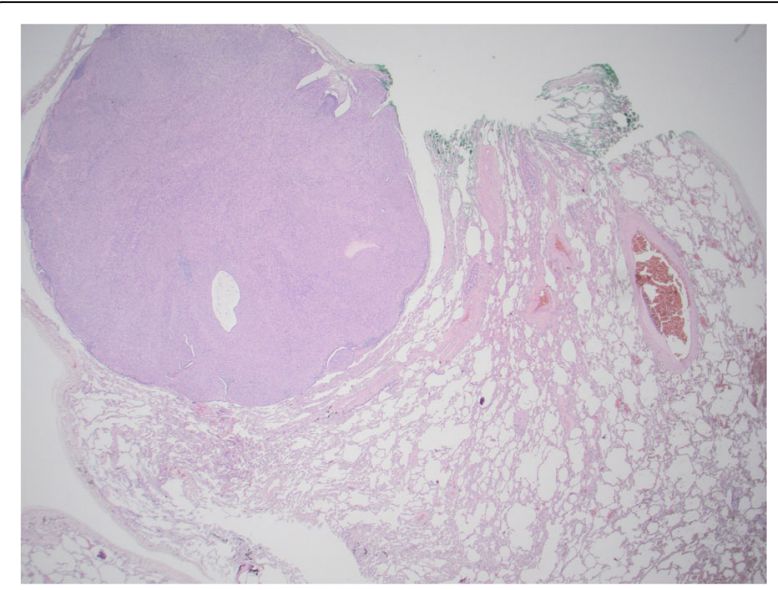

Fig. 2 Low-power image (magnification $\times 12.5$ ). Staining with haematoxylin and eosin. Well-circumscribed pulmonary BML nodule. Green ink marking the surgical resection margin

underwent a PET scan prior to procedure, due to low suspicion of malignancy in the preoperative multidisciplinary team meeting. None of our patients had a PET scan postoperatively as suspicion of malignant transformation remained low, both clinically and radiologically at follow-up.

All of our patients had a wedge resection for diagnosis; the presence of oestrogen and progesterone receptors were found in $83 \%$ ( 5 patients) and 100\% (6 patients) respectively. The spindle-shaped cells were all found to be positive for desmin and smooth muscle actin. Previous reports have noted low levels of Ki67 activity, a marker for cellular proliferation; however, this was not measured at our centre [13].

Follow-up imaging showed no morphological change or growth in follow-up of five patients; however, one

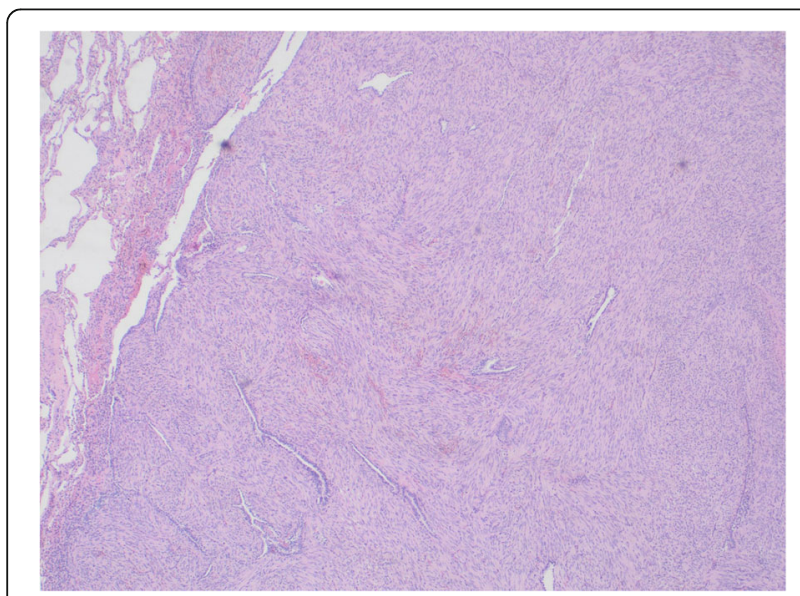

Fig. 3 Higher power image (magnification $\times 100$ ). Staining with haematoxylin and eosin. Cellular spindle cell nodule with entrapped air spaces. Normal lung tissue to the left of the image patient required adjuvant hormonal treatment for nodule regression, due to persistent growth and proximity to structures. There was no consistent follow-up specialty or duration for our patients. Follow-up imaging and interval of imaging were dependant on the clinician in charge of the patient's care.

In our sample of six patients, following successful tissue diagnosis, our patients with BML have a favourable clinical course and outcome with conservative, medical or surgical management. BML caused no complications in any of our patients at follow-up.

With limited knowledge surrounding BML in combination with an ambiguous definition, pathologists require knowledge to recognise a potential BML diagnosis as well as facilities and resources to initiate appropriate analysis of it. The prevalence of BML is unknown; it is questionable if $\mathrm{BML}$ is as rare as the literature suggests or if it is a case of under diagnosis.

\section{Conclusions}

BML is a rare entity usually found in women with a history of uterine leiomyoma and should be considered in the wide variety of differentials for pulmonary nodules.

There are currently no algorithms for the management of BML; however, collating multi-institutional data in prospective centralised databases may help to optimise these strategies.

\section{Abbreviations}

BML: Benign metastasising leiomyoma; LHRH: Luteinizing hormone-releasing hormone; GnRH: Gonadotropin-releasing hormone; VATS: Video-assisted thoracoscopic surgery; H\&E: Haematoxylin and eosin;

FDG: Fluorodeoxyglucose; PET: Positron emission tomography

\section{Acknowledgements \\ None}

\section{Authors' contributions}

This original research idea was that of EJC and AN. EJC and AN performed a literature search. KC and EJC undertook data collection from electronic patient records. SR supervised interpretation of radiological imaging and pathology reports. The first manuscript draft was written by KC and EJC and edited by SR, AN and CJR. CJR obtained microscopic images and provided descriptions, as requested by the associate editor. All authors have read and approved the final manuscript.

\section{Funding \\ None}

\section{Availability of data and materials}

Available on request.

\section{Declarations}

Ethics approval and consent to participate

In line with local and national governance in the UK, the study was registered as a clinical audit at The University Hospitals of Leicester Trust (Registration ID: 10603). Patient data was anonymised, available on request.

\section{Consent for publication}

In line with local and national governance in the UK, the study was registered as a clinical audit at The University Hospitals of Leicester Trust (Registration ID: 10603). Patient data was anonymised, available on request. 


\section{Competing interests}

The authors declare that they have no competing interest.

\section{Author details}

'Department of Thoracic Surgery, Glenfield Hospital, University Hospitals of Leicester Trust, Leicester LE3 9QP, UK. ${ }^{2}$ Department of Histopathology, Leicester Royal Infirmary, University Hospitals of Leicester Trust, LE1 5WW Leicester, UK.

Received: 21 August 2020 Accepted: 13 April 2021

Published online: 29 April 2021

\section{References}

1. Steiner PE (1939) (1939) Metastasizing fibroleiomyoma of the uterus: report of a case and review of the literature. Am J Pathol. 15(1):89-110.7

2. Kayser $K$, Zink S, Schneider T, Dienemann H, André S, Kaltner H, Schüring MP, Zick Y, Gabius HJ (2000) Benign metastasizing leiomyoma of the uterus: documentation of clinical, immunohistochemical and lectin-histochemical data of ten cases. Virchows Arch 437(3):284-292. https://doi.org/10.1007/ s004280000207

3. Miller J, Shoni M, Siegert C, Lebenthal A, Godleski J, McNamee C (2015) Benign metastasizing leiomyomas to the lungs: an institutional case series and a review of the recent literature. Ann Thorac Surg 101(1):253-258. https://doi.org/10.1016/..athoracsur.2015.05.107

4. Chen S, Liu R-M, Li T (2014) Pulmonary benign metastasizing leiomyoma: a case report and literature review. J Thorac Dis 6(6):E92-E98. https://doi.org/1 0.3978/j.issn.2072-1439.2014.04.37

5. Jolissaint JS, Kilbourne SK, LaFortune K, Patel M, Lau CL (2015) Benign metastasizing leiomyomatosis (BML): a rare cause of cavitary and cystic pulmonary nodules. Respir Med Case Reports 16:122-124

6. Barnas E, Ksiazek M, Ras R, Skret A, Skret-Magieroo J, Dmoch-Gajzlerska E (2017) Benign metastasizing leiomyoma: a review of current literature in respect to the time and type of previous gynecological surgery. PLoS One 12(4):1-12

7. Sõritsa D, Teder H, Roosipuu R, Tamm H, Laisk-Podar T, Soplepmann P et al (2018) Whole exome sequencing of benign pulmonary metastasizing leiomyoma reveals mutation in the BMP8B gene. BMC Med Genet 19(1):4-10

8. Irama W, Huang YT, Lee LY, Huang CC, Wan YL (2013) Benign metastasizing leiomyoma of lung. World J Surg Oncol11:1-7.

9. $\mathrm{Ma} \mathrm{H}$, Cao J (2015) Benign pulmonary metastasizing leiomyoma of the uterus: a case report. Oncol Lett 9(3):1347-1350. https://doi.org/10.3892/ol.2 015.2878

10. What is Cancer? https://www.cancer.gov/about-cancer/understanding/whatis-cancer. Accessed 23 September 2020

11. Raposo Ml, Meireles C, Cardoso M, Ormonde M, Ramalho C, Pires M, Afonso M, Petiz A (2018) Benign metastasizing leiomyoma of the uterus: rare manifestation of a frequent pathology. Case Rep Obstet Gynecol. 2018:1-8. https:/doi.org/10.1155/2018/5067276

12. Sawai Y, Shimizu T, Yamanaka Y, Niki M, Nomura S (2017) Benign metastasizing leiomyoma and 18-FDG-PET/CT: a case report and literature review. Oncol Lett 14(3):3641-3646. https://doi.org/10.3892/ol.2017.6609

13. Sawicki M (2015) Pulmonary benign metastasizing leiomyoma from the uterine leiomyoma: a case report. Polish J Radiol. 80:107-110

\section{Publisher's Note}

Springer Nature remains neutral with regard to jurisdictional claims in published maps and institutional affiliations.

\section{Submit your manuscript to a SpringerOpen ${ }^{\circ}$ journal and benefit from:}

- Convenient online submission

- Rigorous peer review

- Open access: articles freely available online

- High visibility within the field

- Retaining the copyright to your article

Submit your next manuscript at $\boldsymbol{\nabla}$ springeropen.com 